\title{
Small Bowel Volvulus due to Mesenteric Lymphatic Malformation: MDCT Demonstration of Counterclockwise Barber Pole Sign
}

\author{
V. Vinay ${ }^{1}$ Venkatraman Bhat ${ }^{1}$ \\ G. K. Pramod ${ }^{1}$ \\ ${ }^{1}$ Department of Imaging Services, Narayana Health, Mazumdar Shaw \\ Medical Centre, Bengaluru, India \\ 2 Department of Pediatric Surgery, Narayana Health, Mazumdar Shaw \\ Medical Centre, Bengaluru, India \\ ${ }^{3}$ Department of Pathology, Narayana Health, Mazumdar Shaw \\ Medical Centre, Bengaluru, India \\ J Child Sci 2017;7:e142-e145.
}

Sanjay Rao ${ }^{2}$

Srinivas Ramaiah ${ }^{3}$

Address for correspondence Venkatraman Bhat, MD, FRCR, Department of Imaging Services, Narayana Health, Mazumdar Shaw Medical Centre, 309, Greenwoods Apartment, Bommasandra, Bengaluru 560099, India (e-mail: bvenkatraman@gmail.com).

\begin{abstract}
Keywords

- mesenteric lymphatic malformation

- small bowel volvulus

- counter clockwise barber pole sign

- CT angiography

Counterclockwise barber pole sign (also known as reverse barber pole sign) is an angiographic sign described in patients with small bowel volvulus due to counterclockwise twisting of mesenteric vessels around the superior mesenteric artery (SMA). The sign was first described in a catheter angiographic examination; however, the concept can be translated to the display methods of computed tomography (CT). This sign can be depicted clearly on multiplanar and three-dimensionally rendered images of multidetector computed tomography (MDCT) examination. In the current report, the reverse barber pole sign is illustrated in a child with partial small bowel volvulus secondary to mesenteric lymphatic malformation. The clinical course, management, and imaging appearances are presented, highlighting the observation of counterclockwise barber pole sign on MDCT imaging. The importance of the sign in identification of volvulus and implications for patient management are emphasized.
\end{abstract}

\section{Introduction}

A 6-year-old female patient presented with a 2-day history of severe periumbilical, colicky abdominal pain. She had six to eight episodes of non-bilious vomiting since the onset of her symptoms, and no bowel movements had been recorded for the preceding 2 days. The patient had two similar previous episodes that resolved spontaneously. The patient had been evaluated at outside health centers on multiple occasions. She was investigated with an abdominal sonography and barium meal follow-through examination. Both examinations were not available for review and were reportedly normal.

On physical examination, vital signs were within normal limits. Abdomen was soft and not distended. Tenderness was present in the umbilical region. No palpable mass was felt. Bowel sounds were normal. Routine laboratory investigations were within normal limits. Abdominal X-rays were performed to exclude bowel obstruction, which showed few non-specific bowel loops with a suggestion of a soft tissue opacity in the central abdomen. There was no definite evidence of bowel obstruction or perforation. Ultrasonography of abdomen showed few fluid-filled cystic spaces in the periumbilical and adjacent supravesical regions. There was evidence of twisting of mesentery along with mesenteric vessels. Multiple prominent lymph nodes were observed in the central abdomen and right iliac fossa. Based on sonographic observation, the possibility of mesenteric lymphatic malformation with small bowel volvulus was considered. There was no peritoneal fluid. No evidence of bowel wall thickening was demonstrated on sonography. Doppler examination suggested parallel, twining configuration of proximal mesenteric vessels at the central abdomen. Evaluation of distal mesenteric vessels and bowel received

April 25, 2017

accepted after revision

September 16, 2017
DOI https://doi.org/

10.1055/s-0037-1607330.

ISSN 2474-5871.
Copyright @ 2017 Georg Thieme Verlag

KG Stuttgart · New York
License terms

(ब) (1) $\circledast$ 
wall was unsatisfactory. Contrast-enhanced multidetector computed tomography (MDCT) examination of abdomen, which was performed to exclude bowel ischemia, demonstrated multiple thin-walled peripherally enhancing fluidfilled cystic spaces in mesentery located in the central abdomen ( - Fig. 1A). There were features of small bowel volvulus with mesenteric twisting (-Fig. 1B). Proximal mesentery showed counterclockwise twisting of more than 360 degrees. There was mesenteric edema ( - Fig. 1B) and narrowing of the superior mesenteric artery (SMA), approximately 3 to $4 \mathrm{~cm}$ from its origin (-Fig. 1C). Maximal intensity projection (MIP) projection and 3-D volumetric display showed the counterclockwise barber pole sign (-Fig. 1C, D). There was no evidence of intestinal obstruction or ischemia.

On diagnostic laparoscopy, a yellowish, fluctuant soft swelling in the mesentery of mid-ileum was seen in association with associated mid-ileal volvulus. The bowel loops were clustered together and appeared pink and healthy. Laparotomy confirmed mesenteric lymphatic malformation and volvulus of small bowel (-Fig. 2A). The lymphatic malformation was acting as the fulcrum of rotation of the mid-ileum. The mass was adherent to adjacent ileal segments, needing resection of the affected bowel segment, and end-to-end anastomosis of the uninvolved bowel. On pathological examination, the small bowel segment measured $9.0 \mathrm{~cm}$ in length with an attached mesentery of $3.5 \mathrm{~cm}$ length. The mesentery had a glistening nodular appearance. On slicing of the mesentery, multiple cysts of variable sizes ranging from 0.2 to $3 \mathrm{~cm}$, containing pale pinkish fluid were noted ( $\mathbf{- F i g . ~ 2 B}$ ). Histopathological examination showed variably sized, thin-walled lymphatic-like channels extending up to muscularis mucosa lined with flattened lining. Lymphatic spaces were seen amidst adipose tissue and fibromuscular stroma ( - Fig. 2C). The features were consistent with a macrocystic lymphatic malformation. The patient's postoperative course was uneventful, and the patient was stable and asymptomatic on follow-up.

\section{Discussion}

Lymphatic malformations are a frequently encountered pediatric disease, representing $6 \%$ of benign tumors in children ${ }^{1,2}$ In pediatric patients, lymphatic malformations are often located in the cervico-facial region and in the axilla. Intra-abdominal localization is less frequent, with a reported incidence of $<5 \%$ of the total., ${ }^{2,3}$ Lesions usually manifest in early childhood and are characterized by a slow, insidious growth. Although the malformation is mostly asymptomatic, some may lead to complications, such as secondary infection, rupture, hemorrhage, volvulus, or intestinal obstruction, particularly when the lesion increases in size and volume. ${ }^{1}$ As a cause of small bowel volvulus, the mesenteric lymphatic malformations constitute a relatively uncommon entity. ${ }^{4,5}$ When small bowel volvulus is encountered, it is a surgical emergency and needs prompt diagnosis and management. Diagnosis of volvulus in older

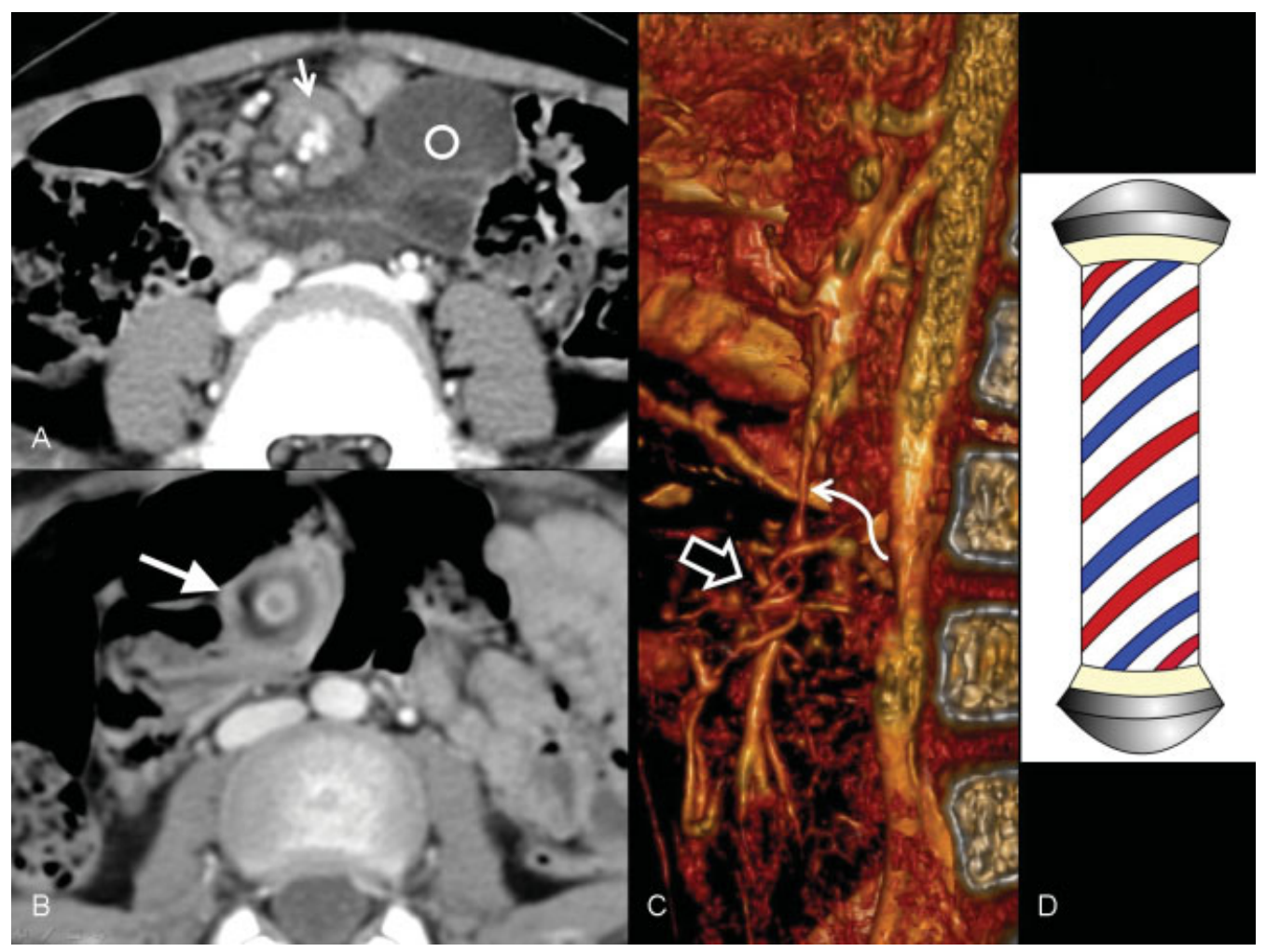

Fig. 1 (A) Axial contrast enhanced CT examination demonstrates cystic lesion in the mesentery (circle). There are clusters of mesenteric vessels with adjacent thickened mesentery (arrow). (B) Thickened mesentery around a pedicle is better demonstrated more proximally (arrow). (C) The 3-D angiographic views show barber's pole sign (open arrow) and narrowing of SMA. (D) Picture of a barber's pole with alternating stripes. $\mathrm{CT}$, computed tomography; SMA, superior mesenteric artery. 


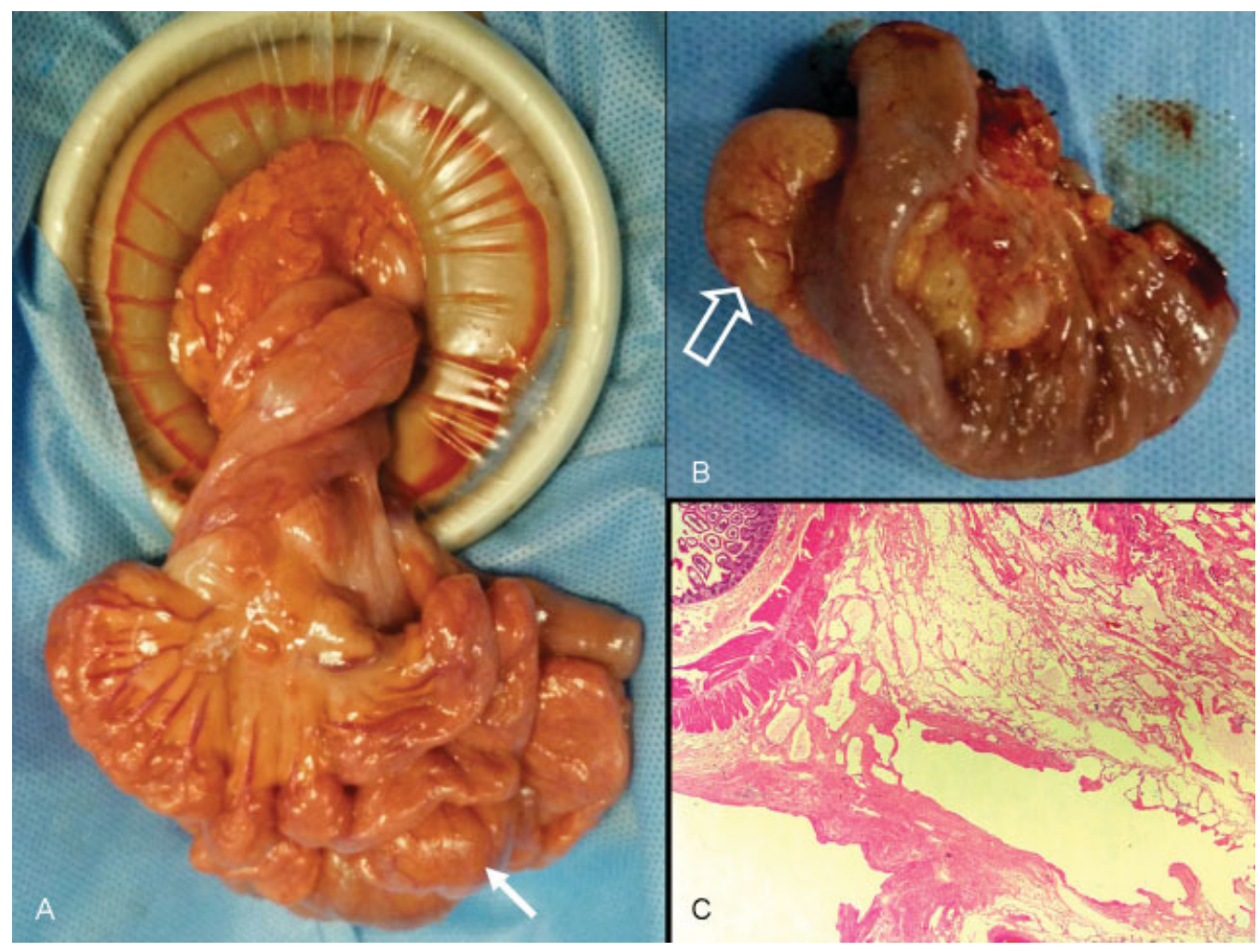

Fig. 2 (A) Operative photograph showing mesenteric volvulus. No evidence of bowel infarction was noted. Specimen photograph (B) shows focal areas of mesenteric cystic masses (open arrow). (C) Photomicrograph (x20) shows part of the small bowel (upper left corner) with adjacent cystic spaces of varying size. Spaces are lined by flattened cells and contain proteinaceous material with lymphocytes. Finding is consistent with lymphatic malformation.

children or adults can be delayed due to non-specific clinical manifestations. It is important to make an early diagnosis to expedite surgical intervention and to avoid intestinal ischemia.

Imaging plays a vital role in defining the diagnosis by demonstrating multiple fluid-filled cysts in the mesentery along with the appearance of small bowel volvulus. Accurate diagnosis can be suspected by sonography ${ }^{6}$ or by CT. While selecting the choice of imaging modalities in children, the concern for radiation needs to be weighed against the need for accurate, unambiguous diagnosis and the promptness with which the final diagnosis can be reached. Due to technical limitations, ultrasound examination often fails to provide accurate information about the presence and extent of bowel ischemia or fails to detect small, peripherally located mesenteric lesions predisposing to volvulus, as exemplified by our patient. In the acute clinical setting, CT examination is often performed as a definitive investigation. MDCT is considered the most useful examination for evaluation of suspected small bowel volvulus. ${ }^{7}$ Radiological interpretation requires, apart from demonstration of the lesion, additional observations, such as signs of cyst rupture, bowel malrotation, volvulus, or bowel ischemia. The presence of volvulus can occasionally be suspected on sonographic examination by observing the relation of the SMA to the vein. Whirled appearance of mesenteric veins around the SMA provides a clue to malrotation. Whirlpool sign is seen in majority of cases of small bowel volvulus on MDCT. It refers to the whirling of branches of superior mesenteric vessels around the main axis of the vessel. The sign basically depicts the axial appearance of the mesentery around the axis of the SMA. Conversely, the "barber pole sign" refers to the lateral profile of the mesentery, along the long axis of mesenteric vessels, when obtained from the contrast-enhanced 3-D rendered dataset of CT. The barber pole sign is due to the multiple stack of intertwining branches of SMA and superior mesenteric vein (SMV) along the path of the mesentery. The barber's pole, which is typically displayed outside barber shops, is characterized by alternating strips of color, in combination of white, red, and blue around a pole. (-Fig. 1D). This imaging sign is coined due to the resemblance of mesenteric vessels to a Barber's pole. MDCT examination is a better suited technique for identifying volvulus and its complications. Interrogation of the axial CT axial dataset, viewed craniocaudally, allows the counterclockwise rotation of the mesentery to be unambiguously appreciated. The sweeping of the mesenteric veins around the SMA is best visualized just beyond the duodenojejunal (DJ) flexure. ${ }^{6}$ Vessels appear like hockey sticks, with SMA in the position of the hook. CT also provides information about mesenteric edema and bowel ischemia. Enhancement pattern of the bowel indicates status of the viability of the bowel loops and possible bowel gangrene. $\mathrm{CT}$ angiography, performed as part of CT evaluation, shows narrowing or occlusion of SMA. Vascular narrowing, as seen in our patient, is an early sign that indicates the potential for bowel infarction. The barber pole sign is occasionally reported in malrotation, complicating mesenteric lymphatic malformation. This sign, also known as 'counterclockwise barber pole sign' was originally described by 
Buranasiri et $\mathrm{al}^{8}$ on an angiographic examination in a patient with counterclockwise malrotation. Reference to the term "reverse barber pole" is made to imply the counterclockwise rotation of the bowel. Hence, it appears appropriate to describe this sign as counterclockwise barber pole sign as the terminology also directly implies the axis of bowel rotation. Although the whirlpool sign and the barber pole sign refer to the same phenomenon viewed from different orientations, the whirlpool sign is more often seen in the proximal mesentery, while the barber pole sign can be detected anywhere along the mesenteric axis. Comparison of the exact incidence of both signs is difficult as there are not many large series reported in the literature. One report evaluating mesenteric volvulus with CT reported the presence of the whirlpool sign in more than half of the cases. No specific reference was made regarding the barber pole sign. ${ }^{7}$ Description of the barber pole sign in association with mesenteric lymphatic malformation is limited to case reports. ${ }^{6}$ Occasionally, this sign is reported on CT angiography examination in adults ${ }^{9}$ and older children. Rarely, this sign is seen in the absence of bowel malrotation. ${ }^{9}$ The 3-D CT angiographic reconstructions and MIP projection of small volumetric dataset of the area of interest enable illustration of this imaging sign most evidently. The length of twisted vessels (barber pole length) will depend upon the extent of rotation and, hence, can vary greatly. Selected, small volume 3-D data processing and surface/MIP rendering around the mesenteric axis will help visualization of a short segment twist in patients with subtle mesenteric rotation.

\section{Conclusion}

A case of mesenteric lymphatic malformation presenting with volvulus of small bowel is described. The presence of "counterclockwise barber pole sign" was shown in the reconstructed MDCT imaging datasets, thereby substantiating the diagnosis. The basis of this not so well-known sign is presented, and we discuss how it differs from a more classical sign, the "whirlpool sign' of mesenteric volvulus. The importance of this sign is highlighted as a means of prompt appreciation of signs of volvulus and in the prediction of bowel ischemia secondary to vascular compression. We also discuss the role of ultrasonography and MDCT in the clinical context of mesenteric volvulus.

\section{Note}

Consent from the guardian for publication was obtained.

Funding

None.

\section{Conflict of Interest}

None.

Acknowledgments

The authors acknowledge the valuable input and contributions from Dr Gautham Shagrith and Dr Vimal Raj for their contributions toward the work. Special thanks to Dr Varun Bhat for reviewing the work and providing constructive suggestions.

\section{References}

1 Garcelan-Trigo JA, Tello-Moreno M, Rabaza-Espigares MJ, Talavera-Martinez I. Barber pole sign in CT angiography, adult presentation of midgut malrotation: a case report. Iran J Radiol 2015; 12(03):e17853

2 Francavilla ML, White CL, Oliveri B, Lee EY, Restrepo R. Intraabdominal lymphatic malformations: pearls and pitfalls of diagnosis and differential diagnoses in pediatric patients. Am J Roentgenol 2017;208(03):637-649

3 Kim SH, Kim H-Y, Lee C, Min HS, Jung S-E. Clinical features of mesenteric lymphatic malformation in children. J Pediatr Surg 2016;51(04):582-587

4 Lilia BH, Rym K, Ali G, et al. Abdominal cystic lymphangioma complicated by mesenteric volvulus: a case report. Tunis Med 2014;92(05):356-358

5 Jang JH, Lee SL, Ku YM, An CH, Chang ED. Small bowel volvulus induced by mesenteric lymphangioma in an adult: a case report. Korean J Radiol 2009;10(03):319-322

6 Singh N, Singh R, Maheswari U, Aga P. Primary mesenteric lymphangioma in a young adult with intestinal malrotation and 'counter-clockwise barber pole sign'. BMJ Case Rep 2013;2013: bcr2013008994

7 Feng ST, Chan T, Sun CH, et al. Multiphasic MDCT in small bowel volvulus. Eur J Radiol 2010;76(02):e13-e18

8 Buranasiri SI, Baum S, Nusbaum M, Tumen H. The angiographic diagnosis of midgut malrotation with volvulus in adults. Radiology 1973;109(03):555-556

9 Clark P, Ruess L. Counterclockwise barber-pole sign on CT: SMA/ SMV variance without midgut malrotation. Pediatr Radiol 2005; 35(11):1125-1127 